\title{
Synchronous Mucinous Borderline Tumor of the Ovary and Low-Grade Appendiceal Mucinous Neoplasm
}

\author{
Tsutomu Muramoto, Ryo Koike \\ Department of Obstetrics and Gynecology, Showa University Fujigaoka Hospital, Yokohama, Japan \\ Email: t_muramo@yahoo.co.jp
}

How to cite this paper: Muramoto, T. and Koike, R. (2021) Synchronous Mucinous Borderline Tumor of the Ovary and LowGrade Appendiceal Mucinous Neoplasm. Open Journal of Obstetrics and Gynecology, 11, 794-803.

https://doi.org/10.4236/ojog.2021.116073

Received: May 17, 2021

Accepted: June 20, 2021

Published: June 23, 2021

Copyright $\odot 2021$ by author(s) and Scientific Research Publishing Inc. This work is licensed under the Creative Commons Attribution International License (CC BY 4.0).

http://creativecommons.org/licenses/by/4.0/

(c) (i) Open Access

\begin{abstract}
We present a rare case of synchronous mucinous borderline tumor of the ovary and low-grade appendiceal mucinous neoplasm (LAMN). We performed a left adnexectomy to diagnose the left ovarian borderline malignancy and an ileostomy because of the swollen appendix during the operation. It was diagnosed as left ovarian mucinous borderline malignancy and LAMN. LAMN causes peritoneal dissemination, ovarian metastasis, and peritoneal pseudomyxoma. The appendix and ovary are close to each other anatomically and can metastasize if there is a tumor in either. For ovarian mucinous tumors, it is necessary to search the gastrointestinal tract, especially the appendix, as the primary lesion. For appendix tumors, it is necessary to search for the ovary. Since LAMN may be associated with borderline ovarian malignancies, as in this case, there is a possibility of the duplication of tumors when searching for ovarian mucinous tumors as the primary tumor and if ovarian tumors are found. Since LAMN and mucinous ovarian tumors have similar histological features, immunohistochemical staining is useful for their differentiation because they show different immunostaining patterns.
\end{abstract}

\section{Keywords}

Low-Grade Appendiceal Mucinous Neoplasm, Mucinous Ovarian Tumor, Synchronous Tumors, Immunohistochemical Staining

\section{Introduction}

Mucinous borderline ovarian tumors are mild to moderately atypical multilocular cystic tumors containing mucoid material and are the most common of the borderline ovarian tumors. Low-grade appendiceal mucinous neoplasm (LAMN) is histologically benign. However, it could spread to the peritoneum as pseudo- 
myxoma peritonei (PMP) and also take a clinically malignant course, such as metastasis to the ovaries. Ovarian metastases of LAMN closely resemble mucinous ovarian tumors in appearance and clinically. Treatment of LAMN with ovarian metastases and duplicate tumors of LAMN with primary ovarian tumors commonly undergo surgical resection. However, it is important to differentiate them as the surgical procedure for each type is different. We found an appendiceal tumor during surgery for a mucinous borderline ovarian tumor. We suspected ovarian metastasis from the appendiceal tumor or duplicate tumors of the appendix and ovary. We subsequently performed both an adnexectomy and ileostomy. We report an interesting case of duplicate tumors of ovarian mucinous borderline tumor and LAMN.

\section{Case Report}

A 41-year-old Japanese woman was admitted to our department with a history of abdominal bloating and lower abdominal pain. A laparoscopic resection had been previously performed for a left ovarian benign mucinous tumor. Ultrasonography revealed a $25-\mathrm{cm}$-sized multilocular tumor extending to the hypochondrium. A computed tomography (CT) scan of the abdomen and pelvis revealed a cystic tumor in the left ovary $25 \mathrm{~cm}$ in size (Figure 1). There were no findings suggestive of dissemination, swollen lymph nodes, or metastasis to multiple organs. Enhanced magnetic resonance imaging (MRI) revealed a multilocular cyst extending from the upper navel to the inside of the pelvis and a tumor that had a "stained glass" appearance with a septal structure inside showing different signal intensities (Figure 2). There were no measurable solid components in the tumor, and the right ovary was normal in size. Tumor markers were within normal limits, except for CA125 and CEA (CA125 94.9 U/mL) (CEA 11.0 $\mathrm{ng} / \mathrm{mL}$ ). The presumptive diagnosis of the cyst was a left borderline ovarian tumor. She underwent an adnexectomy of the left ovary. We found the right ovary to be normal and found pale yellow ascites and a multilocular mass occupying

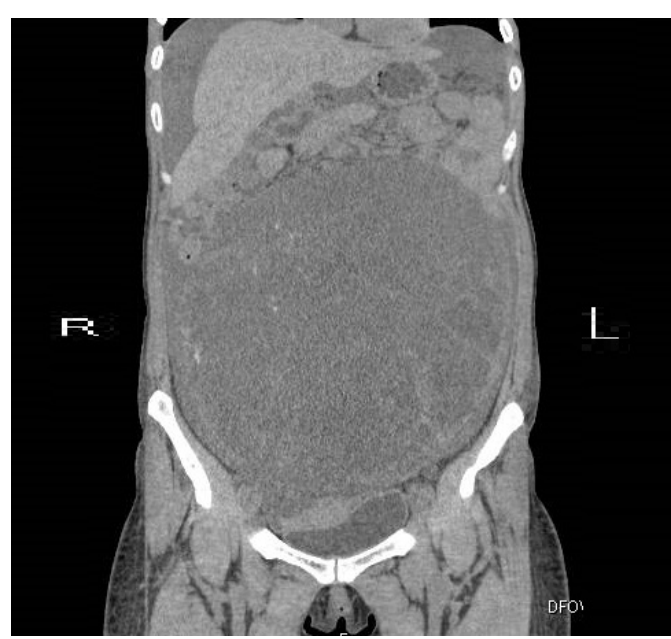

Figure 1. Abdominal CT: a cystic tumor of the left ovary $25 \mathrm{~cm}$ in size extending from the upper navel to the inside of the pelvis. 


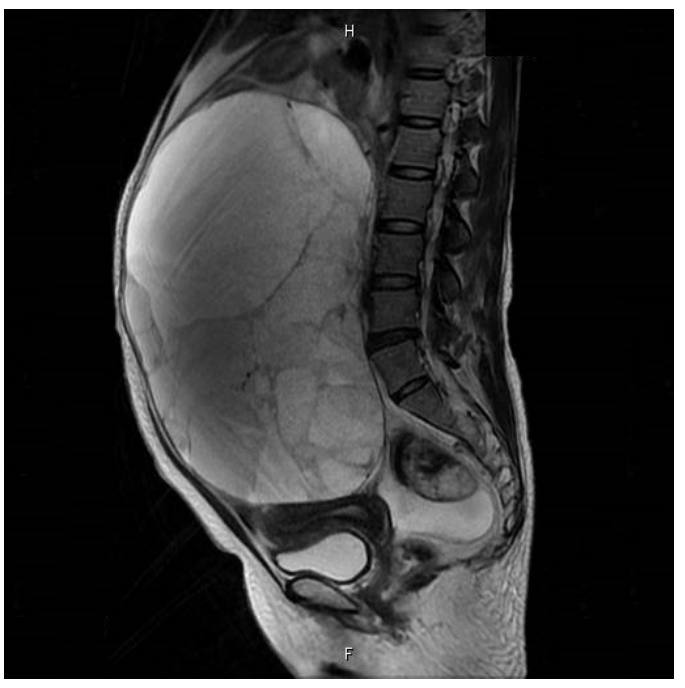

Figure 2. Abdominal MRI: a multilocular cyst extending from the upper navel to the inside of the pelvis and a tumor that had a "stained glass" appearance with a septal structure inside showing different signal intensities.

the abdominal cavity. The tumor had adhered to the pelvic wall and ruptured at the time of removal. The contents of the tumor were yellow and viscous mucus. An intraoperative pathological diagnosis revealed a borderline mucinous tumor of the ovary, but no peritoneal dissemination nor swollen lymph nodes. We found a swollen appendix during the surgery. We considered the possibility of a synchronous tumor of the appendiceal tumor and the ovarian tumor. Alternatively, ovarian metastasis of the appendiceal tumor was also possible. We requested that the surgeons excise the ileocecal. The ileocecal region was resected, and the jejunum and ascending colon underwent layer anastomosis. The operation took 3 hours and 58 minutes. The bleeding, including the tumor content, was 2620 $\mathrm{mL}$ (of which the tumor content was $2000 \mathrm{~mL}$ or more). The postoperative course was unremarkable, with meals started on the third day after the operation. She was discharged 12 days after the procedure.

On macroscopic examination, the ovarian tumor specimen included mucinproducing glandular epithelial cells proliferating in the tubular, papillary, and fused glandular tubes with mild to moderate atypia and obscure or very mild interstitial infiltration (Figure 3). The appendiceal tumor specimen contained a cyst with jelly-like mucus. It also had negative excision margins. A magnified image showed villous proliferative lesions of villous adenoma-like mucinous columnar epithelium with mucous deposits around it with mild stromal infiltration (Figure 4). A frozen section analysis at the time of surgery indicated a mucinous borderline ovarian tumor with a LAMN. Immunohistochemical stains were performed on the paraffin-embedded tissue of ovarian tumors and the appendiceal tumor. Immunohistochemical stains of the ovarian tumor were positive for CK7 and partially positive for CK20 (Figure 5). Immunohistochemical stains of the appendiceal tumor were positive for CK20, and negative for CK7 (Figure 6). 


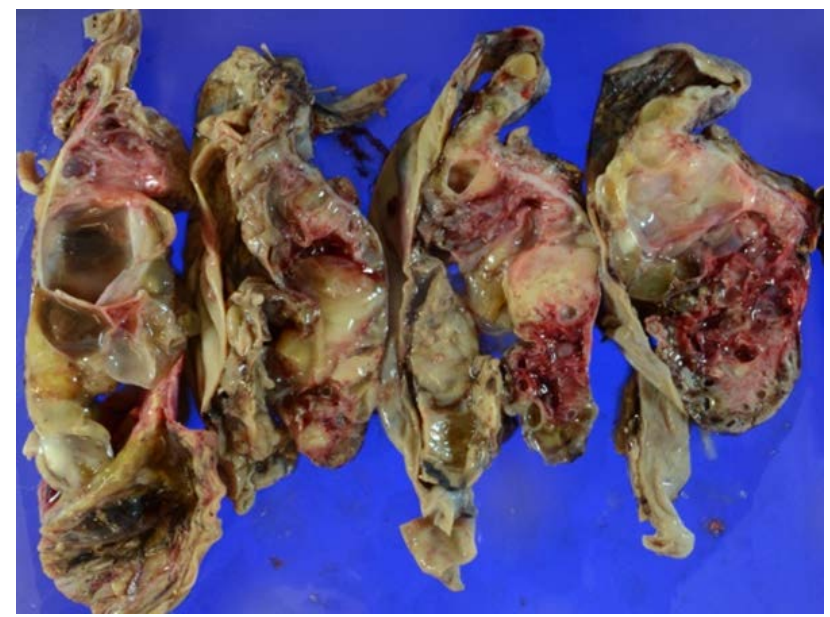

(a)

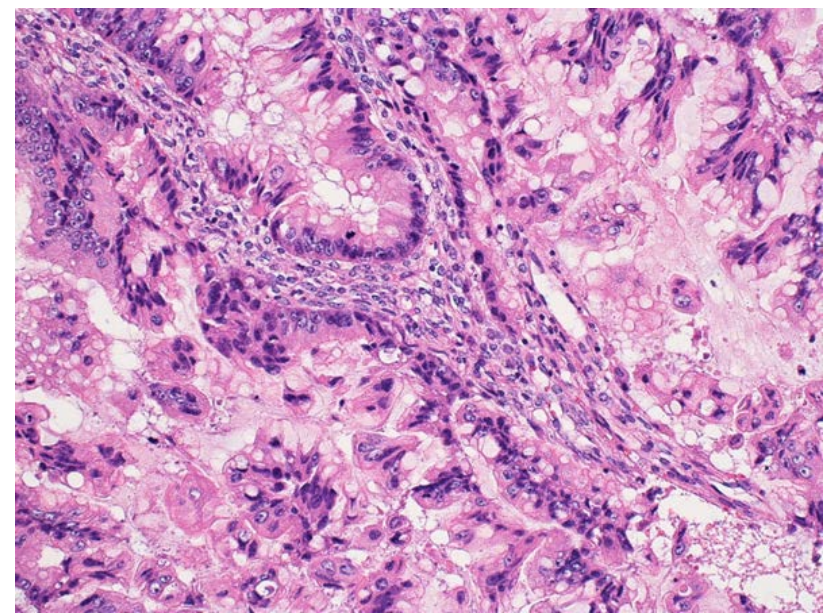

(b)

Figure 3. (a) a polycystic tumor of left ovary; (b) the ovarian tumor included mucinproducing glandular epithelial cells proliferating in the tubular, papillary, and fused glandular tubes with mild to moderate atypia Haematoxylin and eosin stain (H\&E) at $\times 100$ magnification.

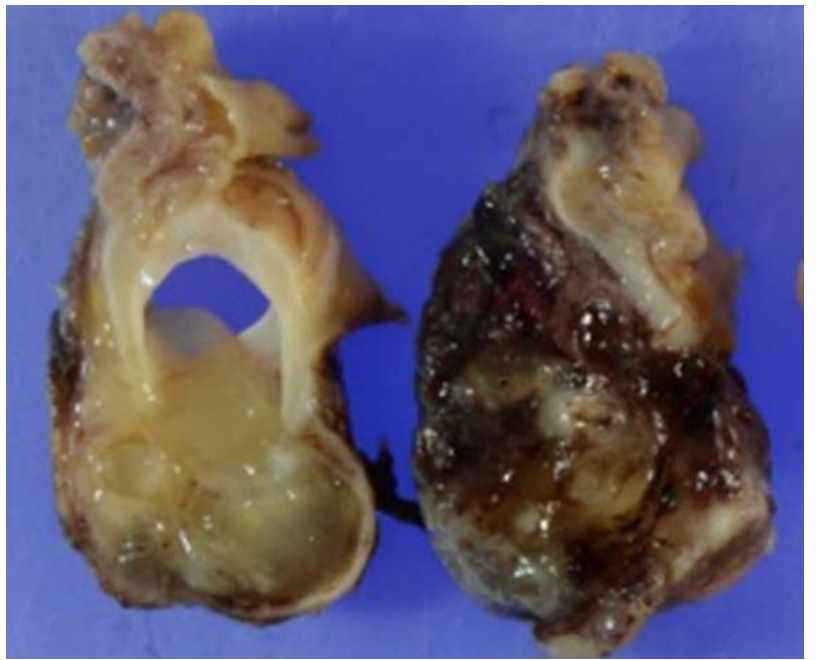

(a) 


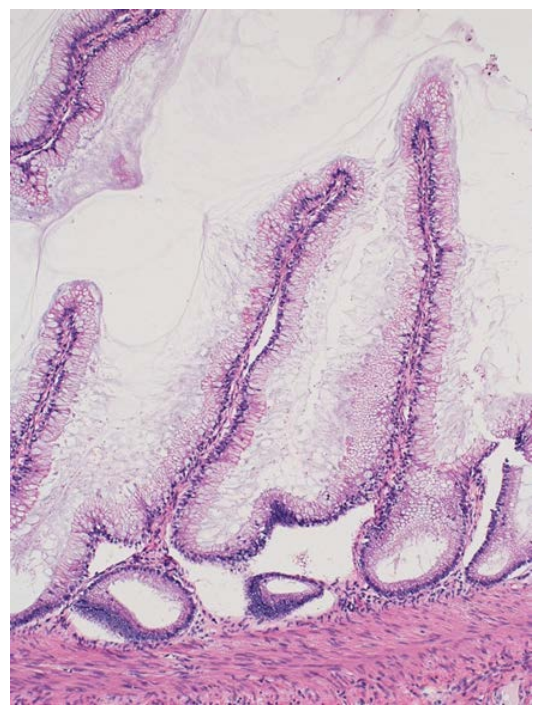

(b)

Figure 4. (a) a cyst with jelly-like mucus of the appendiceal tumor; (b) villous proliferative lesions of villous adenoma-like mucinous columnar epithelium with mucous deposits around it with mild stromal infiltration (H\&E at $\times 200$ magnification).

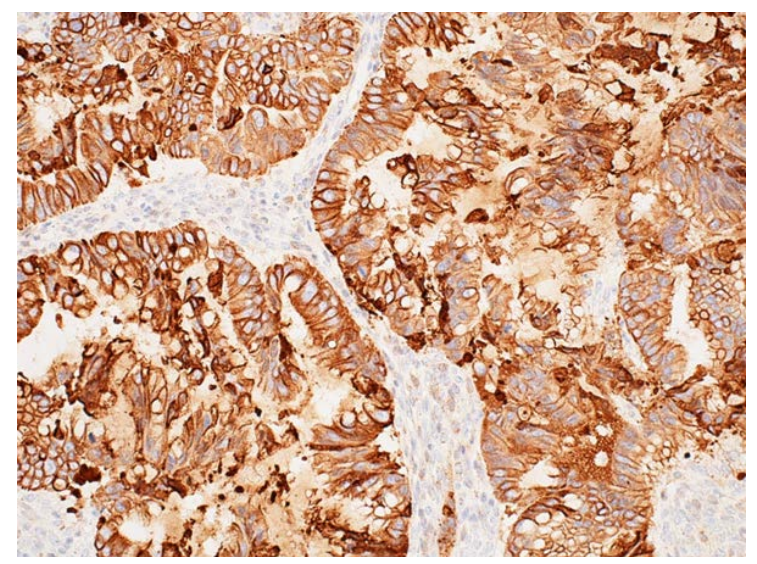

(a)

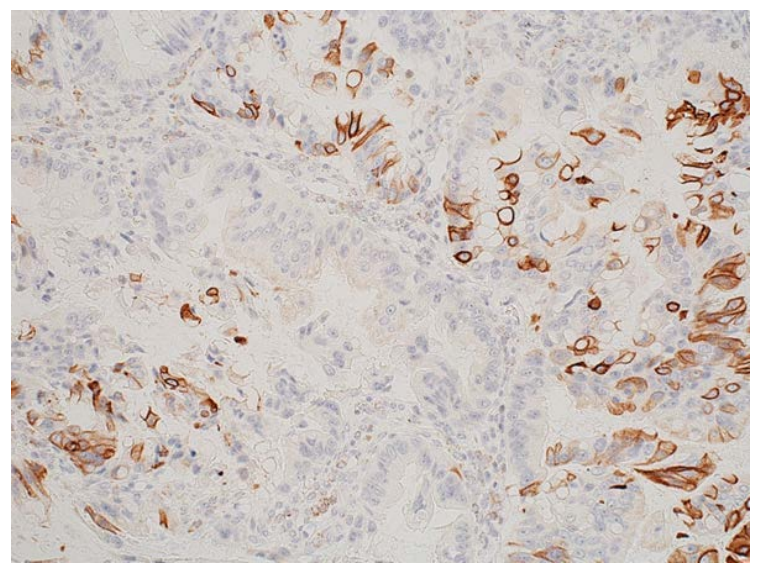

(b)

Figure 5. Immunohistochemical stains of the ovarian tumor were positive for CK7 and partially positive for CK20. (at $\times 200$ magnification). 


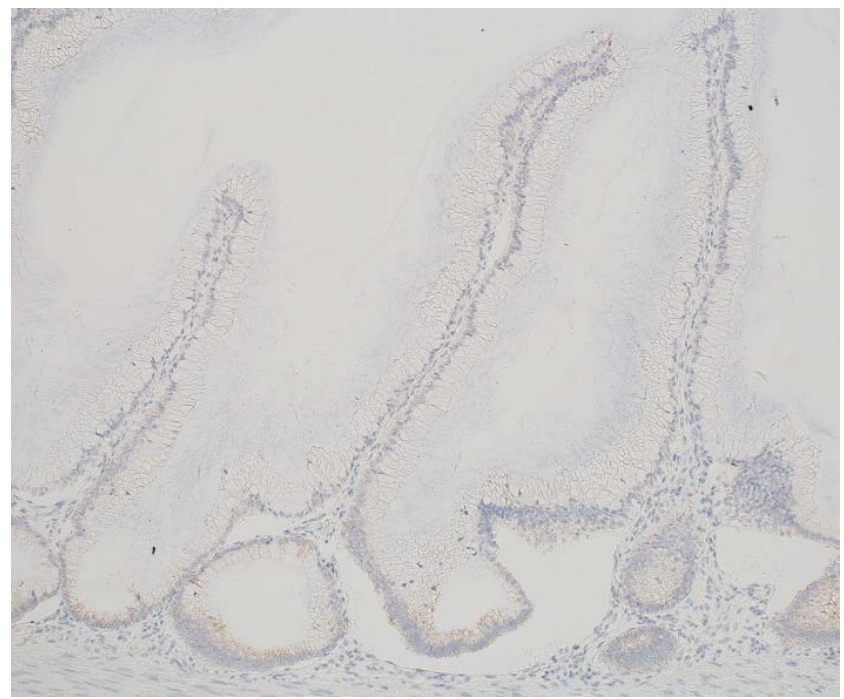

(a)

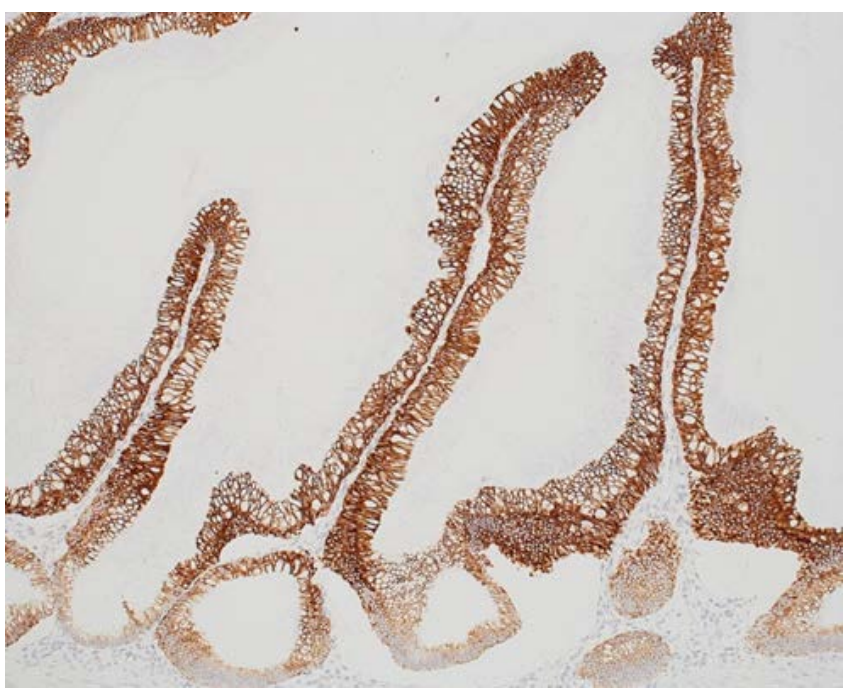

(b)

Figure 6. Immunohistochemical stains of the appendiceal tumor were negative for CK7 and positive for CK20. (at $\times 200$ magnification).

Tumor markers, including CA125 and CEA, were within normal limits after the operation. Because of the borderline mucinous ovarian tumor's pathological diagnosis, she was hospitalized a month later and underwent a total hysterectomy, right adnexal resection, and omentectomy. There were no disseminated lesions in the abdominal cavity, and no metastases were found in the omentum or right ovary. This patient is currently being followed up at an outpatient clinic, and no recurrence has been observed 24 months after the second operation.

\section{Discussion}

LAMN is a low-grade appendiceal mucinous neoplasm, which causes peritoneal dissemination as PMP and ovarian metastasis [1] [2]. Appendiceal tumors could cause symptoms such as right-lower abdominal pain and an abdominal mass [3]. 
Conversely, a swollen appendix is often inconspicuous and is found to be asymptomatic [3]. In our case, an ovarian tumor occupied the abdominal cavity and showed symptoms of abdominal distension and abdominal pain. Appendiceal tumors that presented as dilated cysts in ultrasonography, and MRI or CT were also useful for imaging [2] [4]. It is often difficult to differentiate ovarian tumors and LAMN that are near each other in the pelvis [5] [6]. We could not preoperatively identify the hidden appendiceal tumor close to the ovarian tumor [7] [8]. The treatment of appendiceal tumors, including LAMN, is surgical interventions similar to the treatments for colon cancer [2]. Although there is no clear standard on the excision of a lesion through a surgical procedure, it has been reported that mucinous cystadenoma can develop at the appendectomy stump [7]. Therefore, in our case, ileostomy instead of appendectomy, was required to secure a surgical margin. Although it has been reported that a lymph node dissection can be performed, the possibility of the lymph node metastasis of LAMN is low, and the therapeutic significance of lymph node dissection is unknown [9]. LAMN does not require postoperative chemotherapy. PMP may occur due to tumor wall rupture or peritoneal dissemination [10]. In the case of PMP, tumor weight reduction would need to be performed with the goal of complete resection [10] [11] [12]. The recurrence rate is high, and chemotherapy is also needed as a postoperative therapy. The standard treatment of LAMN with a borderline ovarian tumor, including surgery has not been settled. When considering the possibility of gastrointestinal tumors' metastasis in borderline ovarian mucinous tumors, it is necessary to search the gastrointestinal tract, especially the appendix, for the primary tumor. In our case, we found a swollen appendix during the operation and considered the possibility of an appendix tumor from metastatic ovarian disease or a synchronous tumor with an appendix tumor. Since no mucus accumulation or peritoneal dissemination was observed in the abdominal cavity, and complete resection of the tumor with LAMN without PMP was performed, we expected a good prognosis.

The ileocecal region was resected to achieve a complete resection of the appendiceal tumor. Since there were no swollen lymph nodes or wall rupturing of the appendiceal tumor, the dissection of the lymph node was omitted. The appendix and ovary are close to each other anatomically, which causes problems regarding the spread of inflammation or metastasis of tumors [8]. When the appendiceal tumor metastasizes to the ovary, this often occurs in the right ovaries, which are near the appendix in anatomy. However, these tumors may also metastasize to the left ovaries [12] [13]. It is important to distinguish between LAMN ovarian metastases and overlapping LAMN and borderline ovarian tumors because they would have to undergo different surgical procedures. The treatment of LAMN with ovarian metastasis is ileostomy and metastatic tumor resection. The basic surgical procedure for borderline ovarian malignancies is total hysterectomy, bilateral salpingo-oophorectomy, and omentectomy. Treatments of appendiceal tumors include tumor resection or appendectomy if the tumors are not malignant. If there is no swelling of the lymph node, intraperito- 
neal dissemination, or preoperative tumor wall rupture, there is little possibility of the tumor causing PMP or lymph node metastasis. In the case of PMP or lymph node metastasis, there is a risk of postoperative recurrence, and, thus, ileocecal resection is required for complete resection rather than an appendectomy. In our case, there was synchronous borderline ovarian tumor and LAMN. Thus, total hysterectomy, right adnexal resection, and omentectomy were performed later. On appendiceal tumors and ovarian tumors, there are many reports of ovarian tumors from appendiceal tumor metastasis and appendiceal tumors from ovarian tumor metastasis. Most cases of PMP show lesions both in the ovaries and appendix. However, reports of synchronous tumors of LAMN and ovarian tumors are rare [11].

Regarding the differentiation between LAMN and mucinous ovarian tumor, the histology of LAMN and mucinous ovarian tumor are very similar, and immunohistochemical staining is useful for differentiating between synchronous and metastatic ovarian tumors. LAMN is CK20-positive by immunohistochemical staining [14]. When LAMN metastasizes to the ovary, the metastatic tumor shows the same staining pattern as LAMN. Ovarian mucinous borderline tumors are diffusely positive for CK7. In our case, immunohistochemical staining showed different patterns between the ovarian tumor and LAMN. The diagnosis was a synchronous tumor of borderline ovarian mucinous tumor and LAMN. Although the pathogenic mechanism is unknown, LAMN may be associated with borderline ovarian tumors even in the absence of PMP, as in our case. In addition to searching for the appendix as the primary lesion during surgery for ovarian mucous tumors, if an appendix tumor is found, in addition to appendiceal metastasis of the appendiceal tumor and the appendiceal metastasis of the ovarian tumor, the possibility of synchronous appendiceal and ovarian tumor should also be considered.

\section{Conclusion}

We experienced a rare case of a synchronous tumor of a mucinous borderline malignant tumor and LAMN. When performing surgery for an ovarian mucinous tumor, it is necessary to search the digestive tract, especially the appendix, for the primary lesion. Furthermore, the possibility of duplicative tumors of the appendix should also be considered. LAMN may be associated with borderline ovarian tumors. Since the surgical procedures between LAMN ovarian metastases and LAMN and ovarian borderline tumors differ, it is important to differentiate between the two. Although the histology of LAMN and ovarian mucinous borderline tumors are very similar, immunohistochemical staining is useful for differentiation because their immunostaining patterns are different.

\section{Authors' Contribution}

Tsutomu Muramoto-diagnosis and treatment, corresponding author.

Ryo Koike-assistance of diagnosis and treatment.

Koike gave Muramoto advice in preparing the dissertation considerations. 


\section{Authors' Statement}

There is no conflict of interests which occurs when the authors remain in a financial or personal relationship which unjustly affects his actions associated with the publication of the manuscript.

Any possible relationship(s) of the author(s) with the party/parties interested in the publication of the manuscript are revealed in the text of the article.

The manuscript has not been published in or submitted to any other journal.

No source of financing.

The patient had given her own informed consent for the case report to be published.

\section{Conflicts of Interest}

The authors declare no conflicts of interest regarding the publication of this paper.

\section{References}

[1] Hajiran, A., Baker, K., Jain, P. and Hashmi, M. (2014) Case of an Appendiceal Mucinous Adenocarcinoma Presenting as a Left Adnexal Mass. International Journal of Surgery Case Reports, 5, 172-174. https://doi.org/10.1016/j.ijscr.2013.12.008

[2] Misdraji, J. (2010) Appendiceal Mucinous Neoplasms: Controversial Issues. Archives of Pathology \& Laboratory Medicine, 134, 864-870. https://doi.org/10.5858/134.6.864

[3] Panagopoulos, P., Tsokaki, T., Misiakos, E., Domi, V., Christodoulaki, C., Sioutis, D. and Papantoniou, N. (2017) Low-Grade Appendiceal Mucinous Neoplasm Presenting as an Adnexal Mass. Case Reports in Obstetrics and Gynecology, 2017, Article ID: 7165321. https://doi.org/10.1155/2017/7165321

[4] Tirumani, S.H., Fraser-Hill, M., Auer, R., Shabana, W., Walsh, C., Lee, F. and Ryan, J.G. (2013) Mucinous Neoplasms of the Appendix: A Current Comprehensive Clinicopathologic and Imaging Review. Cancer Imaging, 13, 14-25. https://doi.org/10.1102/1470-7330.2013.0003

[5] Martins, C.F., Costa, S., Guedes, P. and Rafael, A.F. (2015) Pseudomyxoma Peritonei: A Rare Tumor Mimicking Primary Ovarian Neoplasia. BMJ Case Reports, 2015, 1-3. https://doi.org/10.1136/bcr-2014-209251

[6] Balci, O., Ozdemir, S. and Mahmoud, A.S. (2009) Appendiceal Mucocele Mimicking a Cystic Right Adnexal Mass. Taiwanese Journal of Obstetrics and Gynecology, 48, 412-414. https://doi.org/10.1016/S1028-4559(09)60333-8

[7] Panarelli, N.C. and Yantiss, R.K. (2011) Mucinous Neoplasms of the Appendix and Peritoneum. Archives of Pathology \& Laboratory Medicine, 135, 1261-1268. https://doi.org/10.5858/arpa.2011-0034-RA

[8] Muramoto, T., Okada, A., Mukai, Y., Koike, R., Ikemoto, M., Yoshiizumi, E., Fukutani, R., Okazaki, M., Yamashita, Y., Takenaka, S., Hamada, S., Nakayama, K., Sasaki, Y., Morioka, M., Ogawa, K., Wakabayashi, T., Date, H., Norose, T. and Oike, N. (2018) A Case of Laparoscopic Surgery for Synchronous Phlegmonous Appendicitis and a Right Ovarian Tumor. Journal of The Showa University Society, 78, 289-295. (In Japanese)

[9] Carr, N.J., Cecil, T.D., Mohamed, F., Sobin, L.H., Sugarbaker, P.H., González-Mo- 
reno, S., Taflampas, P., Chapman, S. and Moran, B.J. (2016) Peritoneal Surface Oncology Group International (PSOGI) Modified Delphi Processl. A Consensus for Classification and Pathologic Reporting of Pseudomyxoma Peritonei and Associated Appendiceal Neoplasia: The Results of the Peritoneal Surface Oncology Group International (PSOGI) Modified Delphi Process. The American Journal of Surgical Pathology, 40, 14-26. https://doi.org/10.1097/PAS.0000000000000535

[10] Zhou, F., Chen, X., Li, Y. Huang, L. (2015) Two Independent Primary Mucinous Tumors Involving the Appendix and Ovary Accompanied with Acellular Pseudomyxoma Peritonei. International Journal of Clinical and Experimental Pathology, 8, 11831-11834.

[11] Suh, D.S., Song, Y.J., Kwon, B.S., Lee, S., Lee, N.K., Choi, K.U. and Kim, K.H. (2017) An Unusual Case of Pseudomyxoma Peritonei Associated with Synchronous Primary Mucinous Tumors of the Ovary and Appendix: A Case Report. Oncology Letters, 13, 4813-4817. https://doi.org/10.3892/ol.2017.6079

[12] Guerrieri, C., Franlund, B., Fristedt, S., Gillooley, J.F. and Boeryd, B. (1997) Mucinous Tumors of the Vermiform Appendix and Ovary, and Pseudomyxoma Peritonei: Histogenetic Implications of Cytokeratin 7 Expression. Human Pathology, 28, 10391045. https://doi.org/10.1016/S0046-8177(97)90057-5

[13] Gui, X., Escobar, J., Lee, C.H., Duggan, M.A. and Köbel, M. (2017) Synchronous Ovarian and Appendiceal Mucinous Neoplasms in the Absence of Pseudomyxoma Peritonei. International Journal of Gynecological Cancer, 27, 214-222. https://doi.org/10.1097/IGC.0000000000000871

[14] Vang, R., Gown, A.M., Barry, T.S., Wheeler, D.T., Yemelyanova, A., Seidman, J.D. and Ronnett, B.M. (2006) Cytokeratins 7 and 20 in Primary and Secondary Mucinous Tumors of the Ovary: Analysis of Coordinate Immunohistochemical Expression Profiles and Staining Distribution in 179 Cases. The American Journal of Surgical Pathology, 30, 1130-1139. https://doi.org/10.1097/01.pas.0000213281.43036.bb 